\title{
Pediatric Sized REBOA Catheter Testing in a Pulsatile Aortic Flow Model
}

\author{
Erik S DeSoucy DO ${ }^{1,2}$, A Francois Trappey MD' ${ }^{1}$ Anders J Davidson MD, MAS', \\ Joseph J DuBose MD ${ }^{1,2}$, Timothy K Williams $\mathrm{MD}^{3}, \mathrm{M}$ Austin Johnson MD, $\mathrm{PhD}^{4}$ \\ and Jacob T Stephenson MD ${ }^{1,2}$ \\ 'Department of Surgery, David Grant USAF Medical Center, Travis AFB, CA, USA \\ 2Uniformed Services University of Health Sciences, Bethesda, MD, USA \\ ${ }^{3}$ Wake Forest Baptist Health, Winston-Salem, NC, USA \\ ${ }^{4}$ University of Utah Hospital, Salt Lake City, UT, USA
}

\begin{abstract}
Background: Resuscitative Endovascular Balloon Occlusion of the Aorta (REBOA) in the management of pediatric abdomino-pelvic hemorrhage from trauma or iatrogenic injury is limited by a lack of appropriately sized balloon catheters that can be delivered through a less than 7-Fr sheath.

Methods: We bench tested the occlusion capability of eight commercially available balloon catheters deliverable through 4-Fr, 5-Fr, and 6-Fr sheaths in an anatomic pulsatile flow model of the pediatric aorta with variable luminal diameters $(5 \mathrm{~mm}, 6 \mathrm{~mm}, 7 \mathrm{~mm}, 8 \mathrm{~mm}, 9 \mathrm{~mm}, 10 \mathrm{~mm}$, and $12 \mathrm{~mm}$ ). Inflated balloon migration and the deflated balloon's effect on aortic flow were recorded. The flow chamber was calibrated to an approximate size-appropriate physiologic aortic blood flow.

Results: Seven of the eight devices were able to occlude the test lumen diameter corresponding to their manufactured specifications. Deflated luminal flow restriction in the smallest test lumen was lowest in the Fogarty devices (0-3\%) followed by Cordis (8-10\%) and Numed (14-26\%) devices. The Fogarty devices demonstrated the most distal migration (10-15 mm) followed by Numed (1-5 mm). Device migration was undetectable in the Cordis devices.

Conclusion: There are commercially available balloon catheters, deliverable through smaller than 7-Fr sheaths, which can occlude pediatric sized aortic test lumens in the setting of physiologic pulsatile flow. While the use of these catheters for occlusion represents off-label use, these results will help inform future research, device development, and practice in the field of pediatric REBOA.
\end{abstract}

Keywords: Pediatric REBOA; Trauma, Balloon Occlusion Resuscitation; Pulsatile Flow Model

\section{INTRODUCTION}

Trauma remains the leading cause of mortality for children, and contributes to $30-50 \%$ of deaths for children

\section{Corresponding author:}

Major Erik DeSoucy, USAF, MC, FS, Clinical Investigations Facility, David Grant USAF Medical Center, 101 Bodin Cir, Travis AFB, CA 94535, USA.

Email: erik.s.desoucy.mil@mail.mil

Disclosure: The views expressed in this material are those of the authors and do not reflect the official policy or position of the US Government, the Department of Defense, the Department of the Air Force, the University of Utah, or the University of California.

(C) 2020 CC BY 4.0 - in cooperation with Depts. of Cardiothoracic/ Vascular Surgery, General Surgery and Anesthesia, Örebro University Hospital and Örebro University, Sweden greater than 1 year of age [1]. The majority of deaths occurring within the first hour after presentation to a trauma center are attributable to hemorrhage [2]. Thus, the development of immediately available and rapidly deployable adjuncts to control exsanguinating hemorrhage as a bridge to surgical therapy are essential. For appropriately selected adult patients presenting with abdomino-pelvic hemorrhage, resuscitative endovascular balloon occlusion of the aorta (REBOA) is a promising adjunct to temporize patients until operative source control can be obtained $[3,4]$. Utilizing percutaneous or cut-down vascular access, REBOA catheters can be inserted through the common femoral artery (CFA) into the descending aorta and inflated to obtain either complete or partial occlusion, thereby decreasing distal blood flow and augmenting proximal pressure to the brain and heart. Adaptation of this technology to 
children with hemorrhage from trauma or iatrogenic injury during high-risk operations may be beneficial.

The use of REBOA in pediatric patients remains controversial with only limited evidence. Recent models of pediatric trauma using 20-30 kg swine have demonstrated the feasibility of REBOA in a pediatric population $[5,6]$. Norii et al. published a case series using data from the Japan Trauma Data Bank of 54 patients aged 5-17 years who underwent placement of REBOA for trauma [7]. They demonstrated that these patients had equal survival rates to adult trauma patients who underwent REBOA. While most patients were adolescents, the authors recognized that this early clinical use in children was likely increased with the commercial availability of balloon catheters deployed through a 7-Fr sheath instead of a 12-Fr sheath. While this was a significant improvement in device profile, there remains concern regarding morbidity related to access sheath size in young children.

A "one balloon fits all" approach, as utilized in adults, may not be appropriate in the adaptation of REBOA to the pediatric population given the normal morphometric differences amongst children of different ages [8]. In addition, there is greater potential for iatrogenic vessel injury and loss of blood flow to the distal extremity when placing sheaths with a diameter greater than $50 \%$ of the vessel luminal diameter [9]. Sheaths and balloons of varying size will be necessary until a child is large enough to safely undergo femoral cannulation with a 7-Fr sheath. While purpose-built REBOA catheters have not been developed for the pediatric population, or for deployment through a sheath smaller than 7-Fr, existing balloon catheters designed for alternative uses may be applicable to these patients' anatomy. Utilizing a benchtop flow chamber, we sought to evaluate readily available balloon catheters deployable through 4-Fr, 5-Fr, and 6-Fr sheaths. We evaluated their ability to maintain appropriate occlusion throughout a range of aortic diameters and their impact on baseline flow when fully deflated.

\section{METHODS}

\section{Benchtop Flow Chamber}

Our lab developed a benchtop pulsatile flow chamber in order to test occlusion catheters and devices for other experiments related to REBOA (Figure 1). This consisted of an anatomic central arterial circuit with water propelled by a pump. An intermittent solenoid valve controlled by an Arduino microcontroller (Arduino AG, Somerville, MA) with a potentiometer provided pulsatile flow past the main inflow valve. An adjustable bypass segment was placed above the aortic position to allow simulated collateral flow which was titrated with a Hoffman tubing clamp. An in-line flow meter and monitor (Transonic ME 10 PXN and TS410, Transonic Systems Inc., Ithaca, NY) was placed above the aortic occlusion position and pressure was measured both proximal and distal to the aortic position. Replaceable aortic segments were made from polyvinyl chloride tubing of various internal diameters $(5 \mathrm{~mm}, 6 \mathrm{~mm}, 7 \mathrm{~mm}$, $8 \mathrm{~mm}, 9 \mathrm{~mm}, 10 \mathrm{~mm}$, and $12 \mathrm{~mm}$ ) to approximate different aortic diameters in children. A 21-Fr sheath was used as the circuit access point for device placement.

\section{Flow Chamber Calibration}

For each series of testing, the sized aortic segment was installed and pressure and flow were calibrated to estimated physiologic maximums without an occlusion balloon in place. All balloons were tested at a mean arterial pressure (MAP) of $60 \mathrm{mmHg}$ to mimic the upper range of normal blood pressure with the expectation that hypotensive pressures are less likely to result in balloon occlusion failure. Aortic flow was calibrated based on $50 \mathrm{ml} / \mathrm{kg} / \mathrm{min}$ using the expected upper limit of weight for each aortic diameter. To fully recapitulate human aortic occlusion physiology, the bypass circuit clamp was adjusted so that during periods of complete aortic segment occlusion with a clamp, the distal systolic blood pressure (SBP) was approximately $10 \%$ of the proximal SBP. The calibration parameters for each aortic segment diameter can be found in Table 1 .

\section{Balloon Catheter Testing}

We selected eight devices from three manufacturers (Cordis, Edwards Lifesciences, Numed) deployable through 4-Fr, 5-Fr, and 6-Fr sheaths with balloon diameters of $8 \mathrm{~mm}$ to $12 \mathrm{~mm}$ to be tested in the pulsatile flow chamber (Table 2). During testing, each device was evacuated of air and advanced into the aortic position. Aortic flow with the balloon deflated was noted and used to calculate a percent decrease compared with baseline flow. The balloon was then inflated with water using a computerized syringe pump in $0.05 \mathrm{ml}$ increments until flow past the balloon ceased. Inflation volumes were recorded, and the balloon was left in this position for $1 \mathrm{~min}$ to evaluate for migration or loss of occlusion. Each balloon was observed for migration and any changes in structure or wall apposition during inflation. The Fogarty embolectomy catheter (PN 120804F) only has a balloon channel and was advanced into position without wire guidance. All other devices were inflated with the guidewire extended at least $30 \mathrm{~mm}$ from the tip of the device. If the syringe pump was unable to drive the balloon to occlusion or rupture, manual syringe inflation was attempted. If a device ruptured or failed to occlude the aortic lumen, it was excluded from testing on subsequent aortic sizes.

\section{Ethical Approval and Informed Consent}

Ethical approval was not required. Informed consent was not required. 


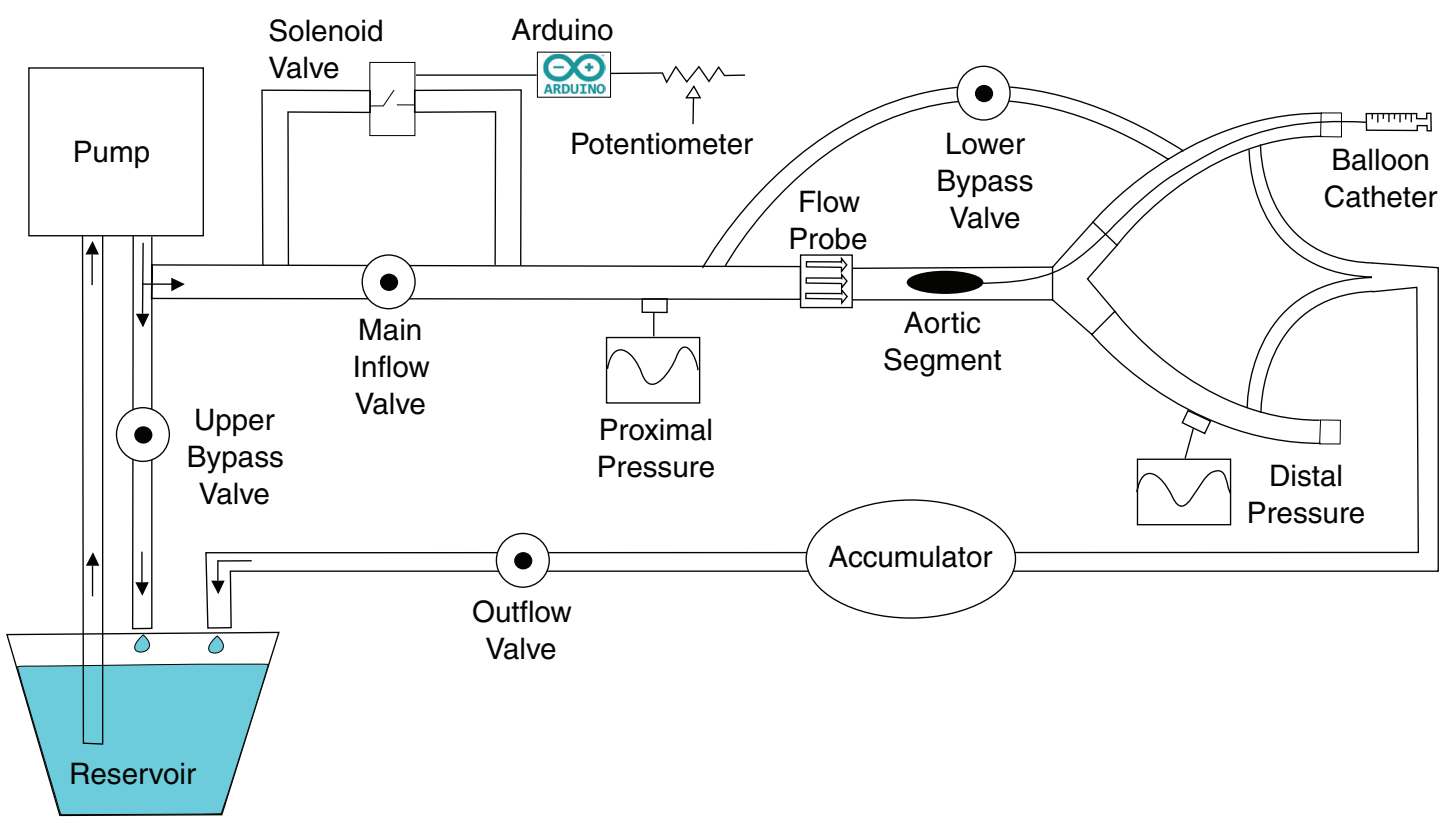

Figure 1 Diagram of pulsatile flow chamber used for occlusion balloon catheter testing.

Table 1 Pulsatile flow chamber settings for aortic occlusion balloon catheter testing.

\begin{tabular}{llllllll}
\hline $\begin{array}{l}\text { Aortic Lumen } \\
\text { Size }(\mathrm{mm})\end{array}$ & $\begin{array}{l}\text { Representative } \\
\text { Weight }(\mathrm{kg})\end{array}$ & $\begin{array}{l}\text { Goal Flow } \\
(\mathrm{ml} / \mathrm{min})^{*}\end{array}$ & $\begin{array}{l}\text { Actual Flow } \\
(\mathrm{ml} / \mathrm{min})\end{array}$ & $\begin{array}{l}\text { MAP } \\
(\mathrm{mmHg})\end{array}$ & $\begin{array}{l}\text { SBP Proximal to } \\
\text { Balloon }(\mathrm{mmHg})\end{array}$ & $\begin{array}{l}\text { SBP Distal to } \\
\text { Balloon }(\mathrm{mmHg})\end{array}$ & $\begin{array}{l}\text { Distal: Proximal } \\
\text { \% Bypass }\end{array}$ \\
\hline 5 & 9 & 450 & 490 & 60 & 95 & 10 & 10.5 \\
6 & 14 & 700 & 700 & 63 & 100 & 10 & 10.0 \\
7 & 18 & 900 & 900 & 62 & 83 & 6 & 7.2 \\
8 & 23 & 1,150 & 1,150 & 59 & 95 & 10 & 10.5 \\
9 & 29 & 1,450 & 1,430 & 60 & 100 & 9 & 9.0 \\
10 & 36 & 1,800 & 1,800 & 60 & 100 & 9 & 10.0 \\
12 & 40 & 2,000 & 2,000 & 60 & 100 & 10 & 10.0 \\
\hline
\end{tabular}

${ }^{*}$ Calculated as representative weight $\times 50 \mathrm{ml} / \mathrm{kg} / \mathrm{min}$. MAP: mean arterial blood pressure; SBP: systolic blood pressure.

\section{RESULTS}

The results from occlusion catheter testing are summarized in Table 3. All but one device (Cordis 4401004S) were able to occlude the lumen commensurate with their manufacture specifications. There were no instances of loss of occlusion after inflation. In three instances, the balloons were inflated beyond manufacturer specifications resulting in device failure with ruptured balloon. The Fogarty 120804F failed at $2.2 \mathrm{ml}$ of inflation, the Fogarty 12TLW804F failed during device removal after occluding the $10 \mathrm{~mm}$ lumen, resulting in tear of the balloon, and the Numed PDC408 ruptured during manual inflation when a $12 \mathrm{~mm}$ occlusion was not obtained using the syringe pump. Table 4 displays the percent decrease in flow when the catheters were fully deflated while positioned in the test lumen. The deflated Fogarty catheters had the least amount of luminal flow restriction $(0-3 \%)$. Deflated Numed devices demonstrated up to a $26.5 \%$ decrease in flow in the smaller lumens with lower baseline flows. As expected, all devices, when deflated, trended toward having less of an impact on flow as lumen diameter and flow increased. Balloon migration was limited to the first $15 \mathrm{~s}$ following occlusion across all devices. The Fogarty devices demonstrated $10-15 \mathrm{~mm}$ of distal migration at the upper limits of testing with significant intraluminal device vibration just prior to occlusion when tested at flow rates over $1,400 \mathrm{ml} / \mathrm{min}$. Numed devices demonstrated $1-5 \mathrm{~mm}$ of migration at the upper limits of testing and the Cordis devices did not migrate.

\section{DISCUSSION}

The use of REBOA has great potential in the management of traumatic abdominal or pelvic hemorrhage and in prophylactic placement for operations with a 
Table 2 Selected occlusion balloon manufacture specifications.

\begin{tabular}{llllll}
\hline Brand & $\begin{array}{l}\text { Part } \\
\text { Number }\end{array}$ & $\begin{array}{l}\text { Introducer } \\
\text { Sheath }(\mathrm{Fr})\end{array}$ & $\begin{array}{l}\text { Balloon } \\
\text { Diameter }(\mathrm{mm})\end{array}$ & $\begin{array}{l}\text { Balloon } \\
\text { Length }(\mathrm{mm})\end{array}$ & $\begin{array}{l}\text { Guidewire } \\
\text { Diameter (inches) }\end{array}$ \\
\hline Fogarty & 120804F & 4 & 9 & 10 & None \\
Fogarty & 12TLW804F & 5 & 9 & 10 & 0.025 \\
Fogarty & 12TLW805F35 & 6 & 11 & 15 & 0.035 \\
Numed & PDC408 & 4 & 10 & 40 & 0.014 \\
Numed & PDC507 & 5 & 12 & 30 & 0.025 \\
Numed & PDC508 & 6 & 12 & 40 & 0.035 \\
Cordis & 44008085 & 5 & 8 & 80 & 0.035 \\
Cordis & 44010045 & 6 & 10 & 40 & 0.035 \\
\hline
\end{tabular}

Table 3 Occlusion balloon catheter occlusion volume in various lumen diameters.

\begin{tabular}{|c|c|c|c|c|c|c|c|c|}
\hline \multicolumn{2}{|c|}{ Balloon Catheter } & \multicolumn{7}{|c|}{ Balloon Occlusion Volume (ml) } \\
\hline Brand & Part Number & $5 \mathrm{~mm}$ & $6 \mathrm{~mm}$ & $7 \mathrm{~mm}$ & $8 \mathrm{~mm}$ & $9 \mathrm{~mm}$ & $10 \mathrm{~mm}$ & $12 \mathrm{~mm}$ \\
\hline Fogarty & $120804 \mathrm{~F}$ & 0.80 & 1.10 & 0.90 & 1.05 & 1.35 & 1.95 & No occlusion \\
\hline Fogarty & 12TLW804F & 0.85 & 0.95 & 1.00 & 1.10 & 1.15 & 1.45 & NT \\
\hline Fogarty & 12TLW805F35 & 0.95 & 0.95 & 1.10 & 1.15 & 1.00 & 1.35 & 1.85 \\
\hline Numed & PDC408 & 1.30 & 1.40 & 1.80 & 2.30 & 2.90 & 4.00 & No occlusion \\
\hline Numed & PDC507 & 1.35 & 1.60 & 1.80 & 2.30 & 2.65 & 3.25 & 4.6 \\
\hline Numed & PDC508 & 1.70 & 1.75 & 2.05 & 2.40 & 3.20 & 3.85 & 5.75 \\
\hline Cordis & 44008085 & 2.80 & 2.90 & 3.35 & 3.85 & No occlusion & NT & NT \\
\hline Cordis & 44010045 & 1.80 & 2.55 & 2.50 & 2.95 & 3.55 & No occlusion & NT \\
\hline
\end{tabular}

NT: not tested due to failure in previous lumen diameter.

high-risk of severe bleeding such as sacrococcygeal or retroperitoneal tumor resection. This potential is tempered by concern for access site complications and the lack of appropriately sized, purpose built pediatric REBOA catheters. The purpose of this study was to evaluate off-the-shelf balloon catheters that could be used for REBOA in pediatric research and practice.

\section{Anatomical Considerations}

If REBOA is to be used in smaller than adult sized children, balloon catheters must be able to be delivered through sheaths smaller than 7 Fr. Alexander et al. identified an increased incidence of loss of lower extremity pulse ipsilateral to the access sheath in children undergoing cardiac catheterization when the sheath outer diameter (OD) was more than $50 \%$ of the arterial luminal diameter (AD) [9] During previous work examining pediatric aortic morphometry and preparing a height based adjunct to the Broselow Tape, we concluded that a child of $122 \mathrm{~cm}$ or 4 feet should have a CFA large enough to accept a 7-Fr sheath while maintaining a $<50 \%$ OD/AD ratio [8]. When time permits, besides simply using height, ultrasound evaluation of the CFA prior to cannulation is prudent and can help with sheath and catheter selection.
With normal aortic anatomy, Zone I extends from the origin of the left subclavian to the level of the celiac axis and Zone III starts at the lowest renal artery and ends at the aortic bifurcation [3]. The length of these REBOA landing zones will decrease as the size of the patient decreases. Properly sized devices should not occlude the renal arteries or the celiac axis when correctly positioned. The two most common adult occlusion balloons, ER-REBOA (Prytime Medical, Boerne, TX) and CODA (Cook Medical, Bloomington, IN) have balloon lengths of $37 \mathrm{~mm}$ and $35 \mathrm{~mm}$. In adults, the median length of Zone I and Zone III are $210 \mathrm{~mm}$ and $97 \mathrm{~mm}$, respectively [10]. In comparison, angiographic measurements of the length of Zone I in 2-year-old children is only $66 \mathrm{~mm}$ and Zone 3 measurement data are lacking [11]. Catheters used for pediatric REBOA in Zone III must be of adequate length to prevent flow past the balloon, but short enough to avoid occlusion of the renal arteries.

\section{Device Considerations}

Ideal aortic occlusion balloons provide complete 360-degree apposition to the vessel wall, have structural longitudinal rigidity to avoid migration, and are deliverable through a sheath that is appropriately sized for the patient's CFA diameter. Due to concerns for migration 
Table 4 Occlusion balloon catheter luminal flow reduction when positioned and fully deflated in various lumen diameters.

\begin{tabular}{|c|c|c|c|c|c|c|c|c|}
\hline \multicolumn{2}{|c|}{ Balloon Catheter } & \multicolumn{7}{|c|}{ \% Decrease in Luminal Flow When Deflated } \\
\hline Brand & Part Number & $5 \mathrm{~mm}$ & $6 \mathrm{~mm}$ & $7 \mathrm{~mm}$ & $8 \mathrm{~mm}$ & $9 \mathrm{~mm}$ & $10 \mathrm{~mm}$ & $12 \mathrm{~mm}$ \\
\hline Fogarty & $120804 \mathrm{~F}$ & 0.0 & 1.4 & 1.1 & 1.7 & 0.7 & 1.1 & 1.0 \\
\hline Fogarty & 12TLW804F & 2.0 & 1.4 & 1.1 & 1.7 & 0.7 & 0.6 & NT \\
\hline Fogarty & 12TLW805F35 & 3.1 & 2.9 & 2.2 & 2.6 & 1.4 & 0.6 & 0.5 \\
\hline Numed & PDC408 & 22.4 & 7.1 & 4.4 & 3.5 & 2.1 & 0.6 & 0.5 \\
\hline Numed & PDC507 & 26.5 & 11.4 & 5.6 & 4.3 & 2.8 & 1.7 & 2.0 \\
\hline Numed & PDC508 & 14.3 & 17.1 & 4.4 & 4.3 & 3.5 & 2.2 & 2.0 \\
\hline Cordis & $4400808 S$ & 8.2 & 8.6 & 5.6 & 8.7 & 4.2 & NT & NT \\
\hline Cordis & 44010045 & 10.2 & 10.0 & 4.4 & 4.3 & 3.5 & 2.2 & NT \\
\hline
\end{tabular}

NT: Not tested due to failure in previous lumen diameter.

and "flipping" of the balloon, we primarily selected "over the wire" balloon devices for testing. In addition, balloon catheters should have a low profile to not only allow passage through a smaller sheath, but also to limit the effects on flow if the balloon is positioned prophylactically and kept deflated. Fogarty devices demonstrated the lowest impact on baseline flow when deflated but were the most apt to migrate when inflated at higher flow rates. Numed devices had less migration and the largest balloon diameter for each of the introducer sheaths tested, but demonstrated the largest decrease in baseline flow when deflated in small vessels with low flow. It is important to note that water was used in the pulsatile flow model which is less viscous than blood. Balloon catheters with significant deflated luminal flow restriction in our model would be expected to have a compounded effect on flow in-vivo.

Balloon compliance plays an important role in occlusion catheters; the most common REBOA catheters (ER-REBOA and CODA) utilize compliant or semicompliant balloons. While material specifications are proprietary to each manufacture, we are able to comment on the general compliance of the devices tested. The Fogarty catheters, which are typically used as embolectomy catheters, demonstrated compliance similar to that of a Foley catheter balloon with a deflated profile, which most closely approximates the shaft of the catheter without any wrinkles in balloon material. This is good for limiting flow obstruction while deflated, but the balloon lengths were small leading to short segment lumen contact and more migration during testing. The Numed catheters tested are designed for pediatric valvuloplasty and as such are somewhat less compliant than the Fogarty balloons. The overall inflated profile more closely approximated the ER-REBOA with good apposition of the lumen walls and minimal migration. This profile resulted in a $14-26 \%$ decrease in flow when deflated in our smallest aortic model. The Cordis catheters used are intended for percutaneous transluminal angioplasty and are specifically designed to be non-compliant. These were the stiffest balloons tested and while they performed well with minimal decrease in aortic flow while deflated and boasted the highest burst pressures, their non-compliant materials may make them more prone to injuring a small vasoconstricted aorta. For the purpose of animal model research, we selected Fogarty balloon catheters for our pediatric swine hemorrhage model and occlusion tolerance studies and would select a similarly designed catheter for clinical applications when indicated.

Table 5 lists the tested catheters by introducer sheath size and their ability to occlude lumens of various sizes and may be used as a guide for catheter selection when these or similar devices are available. The diameter and access sheath size of the commonly used adult occlusion balloon catheters are included for comparison. While these were not tested in our pediatric sized aortic lumens, they have performed well in adult sized conduit at greater pressures and would be expected to provide satisfactory occlusion in smaller lumens.

\section{Limitations}

There are several limitations in this in-vitro analysis of occlusion balloon performance. The tubing used in the replaceable aortic segment is less complaint than that of the young human aorta and lacks the vasoconstriction and vasodilation expected in response to shock and endoluminal manipulation. The test duration for each device was limited to $1 \mathrm{~min}$ of occlusion. In a static inorganic model this would not be expected to change device dynamics after the initial loading of the balloon and catheter body which occurred within $15 \mathrm{~s}$ in our testing. In a dynamic in-vivo model, we would anticipate increased potential for migration due to changes in proximal blood pressure, aortic compliance, and catheter body loading during patient movement. In our model we did not explore partial REBOA which is used to mitigate the metabolic effects of complete occlusion. 
Table 5 Select balloon catheter specifications and occlusion capabilities organized by sheath size.

\begin{tabular}{|c|c|c|c|c|c|c|c|c|c|c|}
\hline \multicolumn{4}{|c|}{ Balloon Catheter Characteristics } & \multicolumn{7}{|c|}{ Device Capable of Occlusion? } \\
\hline Brand & Part Number & Sheath (Fr) & $\begin{array}{l}\text { Balloon } \\
\text { Diameter }(\mathrm{mm})\end{array}$ & $5 \mathrm{~mm}$ & $6 \mathrm{~mm}$ & $7 \mathrm{~mm}$ & $8 \mathrm{~mm}$ & $9 \mathrm{~mm}$ & $10 \mathrm{~mm}$ & $12 \mathrm{~mm}$ \\
\hline Fogarty & $120804 \mathrm{~F}$ & 4 & 9 & Yes* $^{*}$ & Yes* $^{*}$ & Yes* $^{*}$ & Yes $^{*}$ & Yes $^{*}$ & Yes*t & No \\
\hline Numed & PDC408 & 4 & 10 & Yesł & Yes & Yes & Yes & Yes & Yes & No \\
\hline Fogarty & 12TLW804F & 5 & 9 & Yes* & Yes* & Yes* & Yes* & Yes* & Yes*† & No \\
\hline Numed & PDC507 & 5 & 12 & Yesł & Yesł & Yes & Yes & Yes & Yes & Yes \\
\hline Cordis & 44008085 & 5 & 8 & Yes & Yes & Yes & Yes & No & No & No \\
\hline Fogarty & 12TLW805F35 & 6 & 11 & Yes & Yes* & Yes* $^{*}$ & Yes $^{*}$ & Yes* $^{*}$ & Yes* $^{*}$ & Yest* \\
\hline Numed & PDC508 & 6 & 12 & Yesł & Yesł & Yes & Yes & Yes & Yes & Yes \\
\hline Cordis & 44010045 & 6 & 10 & Yesł & Yesł & Yes & Yes & Yes & No & No \\
\hline Prytime & ER-REBOA & 7 & 32 & \multirow{3}{*}{\multicolumn{7}{|c|}{$\begin{array}{l}\text { Included for diameter anc } \\
\text { Not tested in this study }\end{array}$}} \\
\hline Cook & CODA 32 & 12 & 32 & & & & & & & \\
\hline Cook & CODA 40 & 14 & 40 & & & & & & & \\
\hline
\end{tabular}

*Balloon fill volume during occlusion is outside manufacture specifications. +Occlusion diameter outside manufacture specifications. $\neq$ Greater than $10 \%$ flow decrease when in position and deflated. (Note: there are no FDA approved devices for REBOA in pediatric patients.)

Partial occlusion may change the action of the balloon catheter, leading to dynamic changes in balloon profile, migration, and vibration. We did not test each catheter over a range of pressures and instead selected $60 \mathrm{mmHg}$ as a baseline MAP above which it is doubtful a patient would need endovascular occlusion. Alteration in balloon dynamics are possible with the augmented proximal pressure associated with aortic occlusion; however, this was not investigated. We tested a limited number of catheters from suppliers that were readily available in the United States. It is possible a superior performing catheter is available that we are unaware of or do not have access to in our market. Finally, each series of tests were performed with the same set of devices that are generally intended to be used once. It is possible that some of the devices failed due to "wear and tear" from being advanced through the sheath several times and may have performed better if a new device was used each time. It was not financially feasible to obtain multiple of the same device for testing. We mitigated device damage by using a 21-Fr access sheath, minimizing contact between the balloon and sheath during withdrawal.

\section{CONCLUSION}

The lack of size appropriate balloon occlusion catheters is a roadblock to the use of REBOA in the pediatric population for the management of life-threatening bleeding after trauma and during high-risk operations. This study demonstrates the occlusion capabilities of eight off-the-shelf catheters, deliverable through 4-Fr, 5-Fr, and 6-Fr sheaths, in a pulsatile flow aortic model. While the use of these catheters for REBOA represents an off-label use, this study helps inform catheter selection, pediatric trauma model research, and device design in the management of pediatric traumatic or iatrogenic hemorrhage.

\section{Ethics Statement}

(1) All the authors mentioned in the manuscript have agreed to authorship, read and approved the manuscript, and given consent for submission and subsequent publication of the manuscript.

(2) The authors declare that they have read and abided by the JEVTM statement of ethical standards including rules of informed consent and ethical committee approval as stated in the article.

\section{Conflicts of Interest}

MAJ and TKW are stakeholders and cofounders of Certus Critical Care Inc. The other authors declare that they have no conflicts of interest .

\section{Funding}

This research did not receive any specific grant from funding agencies in the public, commercial, or not-forprofit sectors. 


\section{Author Contributions}

ED, FT, AJD, JJD, MAJ, TKW, and JS were responsible for the conception and design. ED and FT performed data acquisition. ED performed data analysis and interpretation. ED drafted the manuscript. ED, FT, AJD, JJD, MAJ, TKW, and JS were involved in critical revision of the manuscript. ED has responsibility for the content.

\section{REFERENCES}

[1] National Center for Health Statistics. National Vital Statistics System: Mortality Tables 1999-2015. https:// www.cdc.gov/nchs/nvss/mortality/lcwk3.htm. Accessed 1 October 2020

[2] Kauvar DS, Lefering R, Wade CE. Impact of hemorrhage on trauma outcome: an overview of epidemiology, clinical presentations, and therapeutic considerations. J Trauma. 2006;60(6 Suppl):S3-11.

[3] Stannard A, Eliason JL, Rasmussen TE. Resuscitative endovascular balloon occlusion of the aorta (REBOA) as an adjunct for hemorrhagic shock. J Trauma. 2011; 71(6):1869-72.

[4] Morrison JJ, Galgon RE, Jansen JO, Cannon JW, Rasmussen TE, Eliason JL. A systematic review of the use of resuscitative endovascular balloon occlusion of the aorta in the management of hemorrhagic shock. J Trauma Acute Care Surg. 2016;80(2):324-34.
[5] Yamashiro KJ, Galganski LA, Grayson JK, et al. Resuscitative endovascular balloon occlusion of the aorta (REBOA) in a pediatric swine model: is 60 minutes too long?. J Trauma Acute Care Surg. 2020; In Press doi: 10.1097/TA.0000000000002620.

[6] Yamashiro KJ, Wishy AM, Beyer CA, et al. Resuscitative endovascular balloon occlusion of the aorta (REBOA) in a pediatric swine liver injury model: a pilot study. J Pediatr Surg. 2020;55(2):346-52.

[7] Norii, T, Miyata S, Terasaka Y, Guliani S, Lu SW, Crandall C. Resuscitative endovascular balloon occlusion of the aorta in trauma patients in youth. J Trauma Acute Care Surg. 2017;82(5):915-20.

[8] DeSoucy ES, Trappey AF, Wishy AM, et al. Approximation of pediatric morphometry for resuscitative endovascular balloon occlusion of the aorta. J Endovasc Resusc Trauma Manag. 2019;3(3):97-103.

[9] Alexander J, Yohannan, T, Abutineh I, et al. Ultrasoundguided femoral arterial access in pediatric cardiac catheterizations: a prospective evaluation of the prevalence, risk factors, and mechanism for acute loss of arterial pulse. Catheter Cardiovasc Interv. 2016;88:1098-107.

[10] Stannard A, Morrison JJ, Sharon DJ, Eliason JL, Rasmussen TE. Morphometric analysis of torso arterial anatomy with implications for resuscitative aortic occlusion. J Trauma Acute Care Surg. 2013;75(2 Suppl 2):S169-72.

[11] Steinberg C, Weinstock DJ, Gold JP, Notterman DA. Measurements of central blood vessels in infants and children: normal values. Cathet Cardiovasc Diagn. 1992; 27:197-201. 